SHORT REPORT

\title{
Birth characteristics of offspring and parental diabetes: evidence for the fetal insulin hypothesis
}

\author{
G Davey Smith, J A C Sterne, P Tynelius, F Rasmussen
}

J Epidemiol Community Health 2004;58:126-128

B irth weight of people is related to their risk of noninsulin dependent diabetes mellitus (NIDDM), which may reflect intra-uterine programming of disease risk, or common genetic factors related both to birth weight and to NIDDM risk. The latter has been called the "fetal insulin hypothesis". ${ }^{1}$ Currently there is little direct evidence for this hypothesis, although two studies have shown an inverse association between fathers' risk of diabetes and the birth weight of their offspring. ${ }^{2}{ }^{3}$ Findings with respect to paternal diabetes are of particular interest because they cannot directly reflect an influence of the intra-uterine environment. Conversely, impaired glucose tolerance in pregnant women is associated with greater offspring birth weight, ${ }^{4}$ and as women with impaired glucose tolerance during pregnancy are at an increased risk of developing diabetes ${ }^{5}$ the expectation is of a positive association between offspring birth weight and maternal diabetes risk. This evidence suggests that as time increases after the birth of offspring an inverse association emerges between offspring birth weight and maternal insulin resistance ${ }^{6}$ and diabetes. ${ }^{3}$

\section{METHODS AND RESULTS}

We investigated the association between parental diabetes and offspring birth characteristics using data from the Swedish Medical Birth Register linked to the Swedish Cause of Death Register. Date of birth, sex, birth weight, birth length, and gestational age, together with the mother's and father's unique identity numbers were extracted from the birth register for all 783814 children born in Sweden between 1973 and 1980. The identity numbers of 783340 mothers and 768583 fathers were recorded in the birth register and mortality data up to 31 December 1997 were obtained. Children with implausible birth weight, birth

length, or gestational age (extreme values or highly improbable combinations of two of these variables) were omitted from analyses, and birth weight and birth length were standardised for the sex of the child.

When different children had the same mother or the same father, one child was randomly chosen, to avoid violation of independence assumptions in the analyses. The final datasets consisted of data on 777695 mother-child pairs, with 573659 pairs after selecting one child for each mother. The corresponding numbers for father-child pairs were 763509 and 563 526. For these parent-offspring pairs educational data were available on 339691 mothers and 396986 fathers: $35.2 \%$ of mothers and $38.0 \%$ of fathers had 10 or fewer years of school education while $10.7 \%$ of mothers and $9.1 \%$ of fathers had post-school education. Associations between offspring birth characteristics and parental diabetes mortality were analysed using Cox proportional hazards regression models, with parent's age as the time axis and entry at the child's date of birth. This means that all analyses are controlled for parents' age. Analyses were censored at the earlier of parent's emigration date (when this was known) and 31 December 1997. In analyses controlling for the confounding effects of other birth characteristics birth weight and birth length were grouped at five levels: lowest decile, 2nd and 3rd deciles, 4th and 5th deciles, 6th and 7th deciles, 8th and 9th deciles, and 10th deciles, while gestational age was grouped at 15 levels: 22-26 weeks, 27-28 weeks, 2930 weeks, 31-32 weeks, 33-34 weeks, weekly from 35 to 44 weeks, 45 plus weeks. These groups were also used in graphical displays. Linearity of birth weight effects was checked by including quadratic terms in the regression models.

Over the follow up period there were 106 deaths from diabetes among mothers, and 334 deaths among fathers.

Table 1 Diabetes mortality in relation to birth characteristics of offspring

\begin{tabular}{|c|c|c|}
\hline & \multicolumn{2}{|l|}{ Hazard ratio $(95 \% \mathrm{Cl})$} \\
\hline & Mothers & Fathers \\
\hline $\begin{array}{l}\text { Birth weight } \\
\text { controlling for gestational age } \\
\text { controlling for birth length } \\
\text { controlling for gestational age and birth length } \\
\text { controlling for offspring year of birth }\end{array}$ & $\begin{array}{l}0.48(0.42 \text { to } 0.54) \\
0.73(0.59 \text { to } 0.91) \\
0.70(0.56 \text { to } 0.87) \\
0.92(0.68 \text { to } 1.24) \\
0.47(0.41 \text { to } 0.54)\end{array}$ & $\begin{array}{l}0.89(0.80 \text { to } 0.98) \\
0.86(0.76 \text { to } 0.97) \\
0.89(0.76 \text { to } 1.04) \\
0.84(0.71 \text { to } 1.01) \\
0.89(0.80 \text { to } 0.98)\end{array}$ \\
\hline $\begin{array}{l}\text { Birth length } \\
\text { controlling for gestational age } \\
\text { controlling for birth weight } \\
\text { controlling for gestational age and birth weight } \\
\text { controlling for offspring year of birth }\end{array}$ & $\begin{array}{l}0.54(0.49 \text { to } 0.59) \\
0.61(0.51 \text { to } 0.73) \\
0.65(0.56 \text { to } 0.75) \\
0.56(0.45 \text { to } 0.69) \\
0.54(0.49 \text { to } 0.59)\end{array}$ & $\begin{array}{l}0.88(0.80 \text { to } 0.98) \\
0.85(0.75 \text { to } 0.96) \\
0.89(0.77 \text { to } 1.04) \\
0.85(0.71 \text { to } 1.01) \\
0.88(0.80 \text { to } 0.97)\end{array}$ \\
\hline $\begin{array}{l}\text { Premature delivery } \\
\text { controlling for birth weight }\end{array}$ & $\begin{array}{l}10.71(7.15 \text { to } 16.04) \\
4.18(2.52 \text { to } 6.91)\end{array}$ & $\begin{array}{l}0.92(0.54 \text { to } 1.57) \\
0.70(0.39 \text { to } 1.25)\end{array}$ \\
\hline
\end{tabular}

Hazard ratios (95\% confidence intervals) per standard deviation higher offspring birth weight $(0.536 \mathrm{~kg}$ in maternal analyses and $0.532 \mathrm{~kg}$ in paternal analyses) or birth length $(0.023 \mathrm{~m}$ maternal and $0.023 \mathrm{~m}$ paternal analyses). 
Table 1 shows that there was a strong inverse association between offspring birth weight and maternal diabetes mortality, with a weaker association among the fathers. For maternal mortality, controlling for gestational age substantially attenuated the association with offspring birth weight, (as would be expected given the considerable collinearity between these variables) whereas for fathers controlling for gestational age had little effect. Premature delivery was associated with a greatly increased risk of mortality from diabetes among mothers: this was considerably attenuated, but remained substantial, after controlling for birth weight. For fathers there was no evidence of an association. Controlling for offspring year of birth made little or no difference to the effect estimates.

Repeating these analyses in the samples on which educational data were available produced similar findings, and controlling for education had no material influence (data not shown). Birth length and birth weight were strongly correlated $(r=0.82)$, and controlling for birth length attenuated the association of birth weight with maternal diabetes mortality. Conversely, controlling offspring birth length for birth weight had little effect. In the case of paternal diabetic mortality, associations with birth weight and birth length were essentially equivalent.

For maternal diabetes mortality, there was no evidence $(p=0.89)$ that the crude effect of birth weight was nonlinear, but clear evidence $(\mathrm{p}<0.001)$ that the effect was nonlinear after controlling for gestational age. For paternal mortality, there was no strong evidence of non-linearity
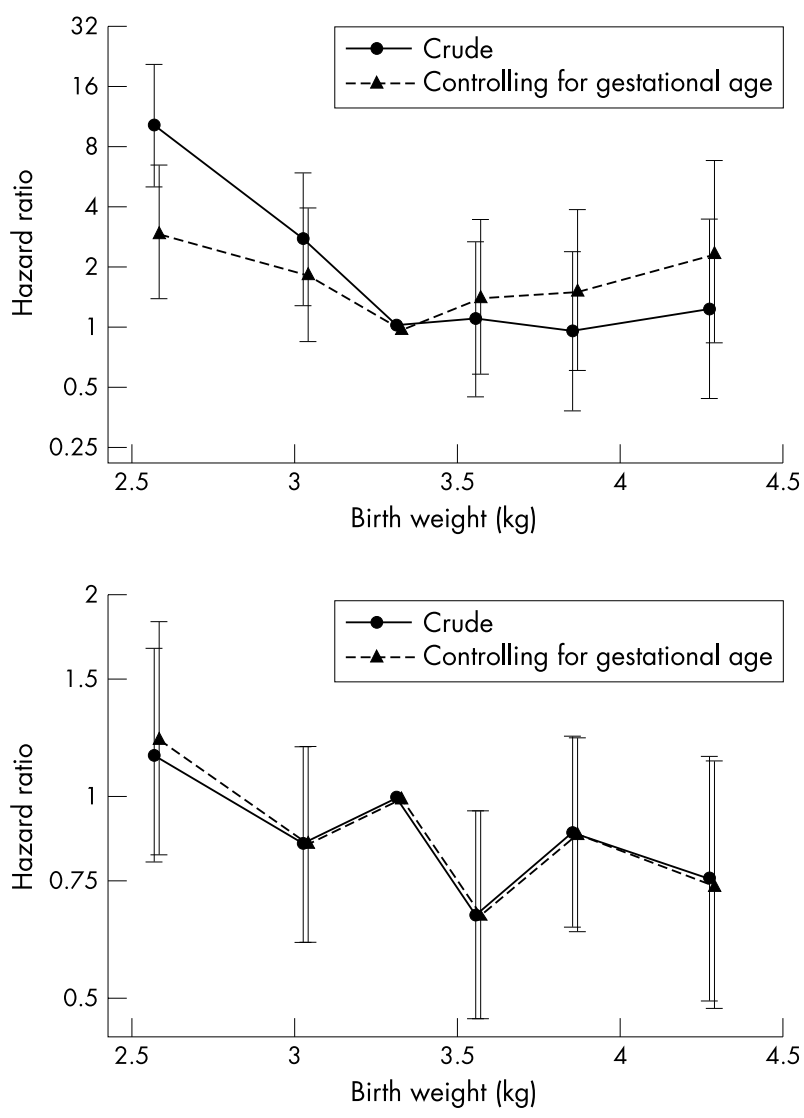

Figure 1 Associations of maternal (upper panel) and paternal (lower panel) diabetes mortality with offspring birth weight, before and after controlling for gestational age. Birth weight is grouped as lowest decile, 2nd and 3rd deciles, 4th and 5th deciles (baseline), 6th and 7th deciles, 8th and 9th deciles, and 10th decile. The horizontal axis shows the median birth weight in each of these groups. either before $(p=0.58)$ or after $(p=0.19)$ controlling for gestational age. Figure 1 shows associations between birth weight groups and maternal and paternal diabetes mortality respectively. After controlling for gestational age, maternal diabetes mortality appeared to be increased at both lower and higher birth weights.

\section{DISCUSSION}

Our results indicate that both intra-uterine and genetic factors lead to associations between parental diabetes risk and offspring birth weight. The inverse association between offspring birth weight and paternal diabetes mortality probably reflects common genetic factors that increase the risk of diabetes in the father and are also related to lower birth weight in the fetus. It is likely that common genetic factors also play a part in the association between maternal diabetes mortality and offspring birth weight, but the greater strength of the inverse association, together with the upturn in risk of maternal diabetes mortality with high offspring birth weight (after controlling for gestational age), indicate that other mechanisms are also involved. Maternal glucose intolerance is associated both with higher offspring birth weight and, later, with increased risk of maternal diabetes, and may account for this upturn. However, offspring of mothers with pre-existing diabetes, or gestational diabetes, are at increased risk of premature birth and growth retardation, ${ }^{7}$ presumably because of the effects of these conditions on intra-uterine environment. This may generate the steep inverse association with low offspring birth weight. Maternal genetic factors related to diabetes risk may also influence offspring birth weight via effects on the intrauterine environment, rather than directly through the segregation of maternal genes to the fetal genome. For example, maternal genes related to greater height of the mother could influence offspring birth weight via an effect on the intra-uterine environment.

Paternal diabetes mortality is not associated with premature delivery of offspring, suggesting that genetic factors shared by the parent and the fetus are not operating in this regard. The strong association between premature birth and maternal diabetes mortality, therefore, probably reflects the influence of an adverse intra-uterine environment provided by the glucose intolerant or frankly diabetic mother.

The differential association of parental diabetes mortality with offspring birth weight or birth length are difficult to interpret, given the strong correlation between birth weight and birth length. There is some suggestion that maternal diabetes mortality is more strongly associated with birth length of offspring than birth weight, whereas this is not the case for paternal diabetes mortality. More data are required on this issue, but the findings could point to an effect of intra-uterine environment on birth length, or to imprinted genes that have a differential effect when transmitted from the mother rather than the father.

The number of diabetic deaths in our study is comparatively small and we have not been able to adjust for some important potential confoundings factors. However, our data provide evidence that both genetic and intra-uterine environmental factors generate associations between parental diabetes risk and offspring outcomes. We suggest that at least some of the association between the birth weight of individuals and their later risk of diabetes may be genetic, and therefore not susceptible to modification by interventions that influence intra-uterine development.

\section{Authors' affiliations}

G Davey Smith, J A C Sterne, Department of Social Medicine, University of Bristol, Bristol, UK 
P Tynelius, F Rasmussen, Department of Epidemiology, Karolinska Institute, Division of Epidemiology, Karolinska University Hospital, Stockholm, Sweden

Correspondence to: Professor G Davey Smith, Department of Social Medicine, University of Bristol, Canynge Hall, Whiteladies Road, Clifton, Bristol BS8 2PR, UK; zetkin@bristol.ac.uk

Accepted for publication 11 May 2003

\section{REFERENCES}

1 Hattersley AT, Tooke JE. The fetal insulin hypothesis: an alternative explanation of the association of low birthweight with diabetes and vascular disease. Lancet 1999;353:1789-92.
2 Lindsay RS, Dabelea D, Roumain J, et al. Type 2 diabetes and low birth weight: the role of paternal inheritance in the association of low birth weight and diabetes. Diabetes 2000;49:445-9.

3 Hyppönen E, Davey Smith G, Power C. Parental diabetes and birth weight of offspring: intergenerational cohort study. BMJ 2003;326:1920.

4 Scholl TO, Sowers M, Chen X, et al. Maternal glucose concentration influences fetal growth, gestation, and pregnancy complications. Am J Epidemiol 2001;154:514-20.

5 Dornhorst A, Rossi M. Risk and prevention of type 2 diabetes in women with gestational diabetes. Diabetes Care 1998;21(suppl 2):B43-9.

6 Lawlor DA, Davey Smith G, Ebrahim S. Birth weight of offspring and insulin resistance in late adulthood: cross sectional survey. $B M J$ 2002;325:359-62.

7 McMahon MJ, Ananth CV, Liston RM. Gestational diabetes mellitus: risk factors, obstetric complications and infant outcomes. J Reprod Med 1998:43:372-8.

\section{$\mathrm{ECHO}$}

\section{Socioeconomic status, occupation, and risk of hospitalisation due to coxarthrosis in} Denmark 1981-99

F Tüchsen, H Hannerz, M V Jensen, N Krause

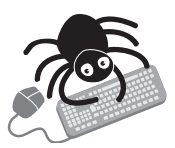

Please visit the Journal of Epidemiology and

Community Health website [www.jech. com] for a link to the full text of this article.
Objectives: To predict the relative risk and time trend in hospitalisation due to coxarthrosis (CA) among groups of different socioeconomic status and occupations in order to test existing aetiological hypotheses.

Methods: Four consecutive cohorts of all gainfully employed Danish men were followed up for CA. Standardised hospitalisation ratios (SHR) and time trends were calculated. Results: Self employed men had an SHR due to CA of 140 (95\% confidence interval (CI) 130 to 151$)$ in $1994-99$. It changed $-1.2 \%$ (95\% CI -2 to -0.4$)$ annually from 1981 to 1999 . Self employed farmers had an SHR of 286 (95\% CI 262 to 313) during 1994-99, increasing 0.14\% a year (95\% CI -0.9 to 1.1) from 1981 to 1999. Unskilled men had an SHR of 121 (95\% CI 113 to 130) in 1994-99. The SHR increased $1.6 \%$ annually (95\% CI 0.7 to 2.4 ) from 1981 to 1999. Employed agricultural workers had an SHR of 189 (95\% CI 158 to 227) from 1994 to 1999. The SHR increased $3.7 \%$ annually (95\% CI 1.2 to 6.2) from 1981 to 1999.

Conclusions: The relative risk of hospitalisation due to CA was consistently high among farmers in four successive analyses spanning an 18 year period. The relative risks remained stable over time despite the fact that the number of farmers decreased. The risk increased among employed workers in agriculture and horticulture over that period, and an increased risk was also found among tractor drivers and a few other occupations.

A Annals of the Rheumatic Diseases 2003;62:1100-1105. 